\title{
Analytic multiplicative cocycles over holomorphic dynamical systems
}

\author{
Tien-Cuong Dinh
}

August 21, 2021

\begin{abstract}
We prove some properties of analytic multiplicative and sub-multiplicative cocycles. The results allow to construct natural invariant analytic sets associated to complex dynamical systems.
\end{abstract}

AMS classification: $37 \mathrm{~F}, 32 \mathrm{H}$

Key-words: multiplicative cocycle, sub-multiplicative cocycle, exceptional set

\section{Introduction}

In this paper we study some sub-multiplicative cocycles over complex dynamical systems. The notion of sub-multiplicative cocycle is classical. It lies at the root of the definition of Lyapunov exponents. In [6] cocycles which are upper semicontinuous with respect to the analytic Zariski topology, were introduced and studied. Applications to holomorphic dynamics were subsequently given in [7, 8]. Our purpose here is to introduce a new approach closer in spirit to [3, 5]. The properties we obtain strengthen former results of Favre. In Section 3 below, we give an application to equidistribution problems of our main theorem, see also [3, 5].

Let $X$ be an irreducible compact complex space of dimension $k$, not necessarily smooth. Let $f: X \rightarrow X$ be an open holomorphic map. Consider the dynamical system associated to $f$ and define $f^{n}:=f \circ \cdots \circ f, n$ times, the iterate of order $n$ of $f$.

Definition 1.1. A sequence of functions $\kappa_{n}: X \rightarrow[1,+\infty[, n \geq 0$, is an analytic sub-multiplicative (resp. multiplicative) cocycle if for any $n, m \geq 0$ and any $x \in X$, we have

1. $\kappa_{n}$ is upper semi-continuous for the Zariski topology on $X$ and $\min _{X} \kappa_{n}=1$;

2. $\kappa_{n+m}(x) \leq \kappa_{n}(x) \kappa_{m}\left(f^{n}(x)\right)\left(\operatorname{resp} . \kappa_{n+m}(x)=\kappa_{n}(x) \kappa_{m}\left(f^{n}(x)\right)\right)$. 
The first property implies that $\kappa_{n}$ is bounded from above and equal to 1 out of a finite or countable union of proper analytic subsets of $X$. Moreover, for any $\delta>1,\left\{\kappa_{n} \geq \delta\right\}$ is a proper analytic subset of $X$ and there is $\delta^{\prime}<\delta$ such that $\left\{\kappa_{n}<\delta\right\}$ is contained in $\left\{\kappa_{n}<\delta^{\prime}\right\}$. The second property defines classical multiplicative and sub-multiplicative cocycles, see [13]. It implies that if $M$ is a constant such that $\kappa_{1} \leq M$, then $\kappa_{n} \leq M^{n}$ for $n \geq 1$.

The new tool we introduce is to define for any analytic sub-multiplicative cocycle $\left(\kappa_{n}\right)_{n \geq 0}$, its extension to the negative integers:

$$
\kappa_{-n}(x):=\max _{y \in f^{-n}(x)} \kappa_{n}(y) .
$$

Since $\left\{\kappa_{-n} \geq \delta\right\}=f^{n}\left\{\kappa_{n} \geq \delta\right\}$, the function $\kappa_{-n}$ is upper semi-continuous in the Zariski sense. Here is our main result.

Theorem 1.2. The sequence of functions $\left[\kappa_{-n}\right]^{1 / n}$ converges pointwise to a function $\kappa_{-}$. Moreover, for every $\delta>1$, the level set $\left\{\kappa_{-} \geq \delta\right\}$ is a proper analytic subset of $X$ which is invariant under $f$ and is contained in the orbit of $\left\{\kappa_{n} \geq \delta^{n}\right\}$ for every $n \geq 0$. In particular, $\kappa_{-}$is upper semi-continuous in the Zariski sense.

Using the function $\kappa_{-}$, we obtain a more uniform version of [6, Th. 2.3.5] for sub-multiplicative cocycles, which is proved to be useful in applications, see Section 3 below.

Corollary 1.3. If $\lim \sup \left[\kappa_{n}(x)\right]^{1 / n} \geq \delta$, then $x$ is sent by some $f^{n}$ to a point in the invariant analytic set $\left\{\kappa_{-} \geq \delta\right\}$. If $\left(\kappa_{n}\right)$ is multiplicative, then $\left[\kappa_{n}\right]^{1 / n}$ converge pointwise to a function $\kappa_{+}$such that $\kappa_{+} \circ f=\kappa_{+}$.

The new and useful property here is that the analytic set $\left\{\kappa_{-} \geq \delta\right\}$ does not depend on $x$. Note that one can extend the study to the case where $f: X \rightarrow X$ is a continuous open map on an irreducible Notherian topological space of finite dimension, see [6, 11]. In particular, the above results hold for open real analytic maps and for open algebraic maps on quasi-projective varieties. We will discuss the case of dominant meromorphic maps on Kähler compact manifolds in Section 3.

Note also that the condition $\min \kappa_{n}=1$ in Definition 1.1 can be replaced by $\kappa_{n} \geq c^{n}$ for some constant $c>0$. Indeed, let $Y$ be a compact analytic set and $b$ a point in $Y$. Consider the map $f^{\prime}: X \times Y \rightarrow X \times Y$ defined by $f^{\prime}(x, y):=(f(x), y)$ and the cocycle $\kappa_{n}^{\prime}(x, y):=1$ if $y \neq b$ and $\kappa_{n}^{\prime}(x, b):=2^{n} c^{-n} \kappa_{n}(x)$. Theorem 1.2 and Corollary 1.3 can be applied to $f^{\prime}$ and $\kappa_{n}^{\prime}$. We deduce analogous results for $\delta>\delta^{*}:=\min \kappa_{-}\left(\right.$in Theorem 1.2 and Corollary 1.3 we have $\delta^{*}=1$ ).

\section{Asymptotic behavior of cocycles}

In this section, we prove the results stated above. Observe that $\left(\kappa_{n l}\right)_{n \geq 0}$ is an analytic (sub-)multiplicative cocycle for $f^{l}$ with $l \geq 1$. We have the following elementary lemmas. 
Lemma 2.1. Let $l \geq 1$ be an integer. Then

$$
\limsup _{n \rightarrow \infty}\left[\kappa_{n l}\right]^{1 /(n l)}=\limsup _{n \rightarrow \infty}\left[\kappa_{n}\right]^{1 / n} \quad \text { and } \quad \liminf _{n \rightarrow \infty}\left[\kappa_{n l}\right]^{1 /(n l)}=\liminf _{n \rightarrow \infty}\left[\kappa_{n}\right]^{1 / n} .
$$

Proof. If $M$ is a constant such that $\kappa_{s} \leq M$ for any $0 \leq s \leq l$, then

$$
\kappa_{m+s}(x) \leq \kappa_{m}(x) \kappa_{s}\left(f^{m}(x)\right) \leq M \kappa_{m}(x) .
$$

We deduce from these inequalities that

$$
M^{-1} \kappa_{(n+1) l} \leq \kappa_{n l+s} \leq M \kappa_{n l}
$$

Hence

$$
\limsup _{n \rightarrow \infty}\left[\kappa_{n l+s}\right]^{1 /(n l+s)}=\limsup _{n \rightarrow \infty}\left[\kappa_{n l}\right]^{1 /(n l)}
$$

for every $0 \leq s \leq l-1$. The first identity in the lemma follows. The last one is obtained in the same way.

Lemma 2.2. Let $l \geq 1$ be an integer. Then

$$
\limsup _{n \rightarrow \infty}\left[\kappa_{-n l}\right]^{1 /(n l)}=\limsup _{n \rightarrow \infty}\left[\kappa_{-n}\right]^{1 / n} \quad \text { and } \quad \liminf _{n \rightarrow \infty}\left[\kappa_{-n l}\right]^{1 /(n l)}=\liminf _{n \rightarrow \infty}\left[\kappa_{-n}\right]^{1 / n} .
$$

Proof. As in Lemma 2.1, we get

$$
\kappa_{m+s}(x) \leq \kappa_{s}(x) \kappa_{m}\left(f^{s}(x)\right) \leq M \kappa_{m}\left(f^{s}(x)\right) .
$$

Therefore, if $a \in X$ and $b \in f^{-m-s}(a)$ are such that $\kappa_{m+s}(b)=\kappa_{-m-s}(a)$, then

$$
\kappa_{-m-s}(a)=\kappa_{m+s}(b) \leq M \kappa_{m}\left(f^{s}(b)\right) \leq M \max _{x \in f^{-m}(a)} \kappa_{m}(x)=M \kappa_{-m}(a) .
$$

We deduce that

$$
M^{-1} \kappa_{-(n+1) l} \leq \kappa_{-n l-s} \leq M \kappa_{-n l}
$$

The lemma follows.

For any irreducible analytic subset $Y$ of $X$, define

$$
\kappa_{n}(Y):=\min _{x \in Y} \kappa_{n}(x) \text { and } \quad \kappa_{-n}(Y):=\min _{x \in Y} \kappa_{-n}(x) .
$$

Since $\kappa_{n}$ and $\kappa_{-n}$ are upper semi-continuous for the Zariski topology, the previous minimums always exist. Moreover, we have $\kappa_{n}(Y)=\kappa_{n}(x)$ and $\kappa_{-n}(Y)=\kappa_{-n}(x)$ for $x \in Y$ outside a finite or countable union of proper analytic subsets of $Y$.

Lemma 2.3. Let $Y$ be a periodic irreducible analytic subset of $X$. Then $\left[\kappa_{n}(Y)\right]^{1 / n}$ converge to a constant $\kappa_{+}(Y)$. Moreover, for every $m \geq 0$, we have $\kappa_{+}\left(f^{m}(Y)\right)=$ $\kappa_{+}(Y)$ and $\lim \inf \left[\kappa_{-n}(Y)\right]^{1 / n} \geq \kappa_{+}(Y)$. If $\kappa_{+}(Y) \geq \delta$ then for every $m \geq 0, Y$ is contained in the orbit of $\left\{\kappa_{m} \geq \delta^{m}\right\}$. 
Proof. Assume that $f^{l}(Y)=Y$ with $l \geq 1$. Using properties of cocycles for a generic point in $Y$, we have

$$
\kappa_{(m+n) l}(Y) \leq \kappa_{m l}(Y) \kappa_{n l}\left(f^{m l}(Y)\right)=\kappa_{m l}(Y) \kappa_{n l}(Y)
$$

Hence, the sequence of $\left[\kappa_{n l}(Y)\right]^{1 /(n l)}$ is decreasing and converges to some constant $\kappa_{+}(Y)$ when $n$ tends to infinity. The first assertion follows from Lemma 2.1 applied to a generic point in $Y$. By definition of $\kappa_{-n}$, since $f^{l}(Y)=Y$, we have $\kappa_{-n l}(Y) \geq \kappa_{n l}(Y)$. This and Lemma 2.2 imply the assertion on $\kappa_{-n}$.

For the second assertion, applying (1) for a generic point in $Y$ or $f(Y)$ yields

$$
M^{-1} \kappa_{n+1}(Y) \leq \kappa_{n}(f(Y)) \leq M \kappa_{n-l+1}\left(f^{l}(Y)\right)=M \kappa_{n-l+1}(Y)
$$

This and the first assertion imply that $\left[\kappa_{n}(f(Y))\right]^{1 / n}$ converge to $\kappa_{+}(Y)$. It follows that $\kappa_{+}(f(Y))=\kappa_{+}(Y)$ and then $\kappa_{+}\left(f^{m}(Y)\right)=\kappa_{+}(Y)$ for every $m \geq 0$.

Assume that $\kappa_{+}(Y) \geq \delta$ and that $Y$ is not contained in the orbit of $\left\{\kappa_{m} \geq \delta^{m}\right\}$ for a fixed $m \geq 0$. Since $Y$ is periodic, $f^{i}(Y)$ is not contained in $\left\{\kappa_{m} \geq \delta^{m}\right\}$ for every $i \geq 0$. We then deduce that $\kappa_{m}\left(f^{i}(Y)\right) \leq \delta_{0}^{m}$ for some constant $\delta_{0}<\delta$. This implies

$$
\kappa_{n m}(Y) \leq \prod_{i=0}^{n-1} \kappa_{m}\left(f^{i m}(Y)\right) \leq \delta_{0}^{n m}
$$

that contradicts the inequality $\kappa_{+}(Y) \geq \delta$.

We have the following proposition.

Proposition 2.4. Let $q$ be an integer with $0 \leq q \leq k-1$. Let $\Omega$ be a dense Zariski open subset of $X$ such that $f^{-1}(\Omega) \subset \Omega$. Assume that $\left\{\kappa_{-n_{0}} \geq \delta^{n_{0}}\right\} \cap \Omega$ is of dimension at most equal to $q$ for some $n_{0} \geq 1$ and some $\delta>1$. Then there is an integer $n_{1} \geq 1$ and an invariant proper analytic subset $\Sigma$ of $X$, possibly reducible, which is empty or of pure dimension $q$, such that

1. $\Sigma$ is contained in the orbit of $\left\{\kappa_{n} \geq \delta^{n}\right\}$ for every $n \geq 0$;

2. $\liminf \left[\kappa_{n}(x)\right]^{1 / n} \geq \delta$ and $\liminf \left[\kappa_{-n}(x)\right]^{1 / n} \geq \delta$ for $x \in \Sigma$;

3. $\left\{\kappa_{-n_{1}} \geq \delta^{n_{1}}\right\} \cap \Omega \backslash \Sigma$ is of dimension $\leq q-1$ if $q \geq 1$ and is empty if $q=0$.

Proof. Let $\widetilde{\Sigma}$ denote the union of the irreducible components of dimension $q$ in the closure of $\left\{\kappa_{-n_{0}} \geq \delta^{n_{0}}\right\} \cap \Omega$. Recall that $\kappa_{-n_{0}}$ is upper semi-continuous and that $\left\{\kappa_{-n_{0}} \geq \delta^{n_{0}}\right\} \cap \Omega$ is of dimension less than or equal to $q$. Hence, there is a real number $1 \leq \delta_{0}<\delta$ satisfying $\kappa_{n_{0}}(V) \leq \delta_{0}^{n_{0}}$ for any irreductible analytic subset $V$ of dimension $\geq q$ of $X$ such that $f^{n_{0}}(V)$ intersects $\Omega \backslash \widetilde{\Sigma}$. We will cover $\widetilde{\Sigma}$ by three analytic sets $\Sigma, \Sigma^{\prime}$ and $\Sigma^{\prime \prime}$.

Define $\Sigma$ as the union of the orbits of periodic irreducible components $Y$ of $\widetilde{\Sigma}$ such that $\kappa_{+}(Y) \geq \delta$. So, $\Sigma$ is invariant, i.e. $f(\Sigma)=\Sigma$. Note that $\Sigma$ is not 
necessarily contained in $\widetilde{\Sigma}$. By Lemma 2.3, we also have $\kappa_{+}\left(f^{i}(Y)\right) \geq \delta$ for every $i \geq 0$ and $\Sigma$ satisfies the first two properties in the proposition. Let $\Sigma^{\prime}$ be the union of the orbits of periodic irreducible components $Y$ of $\widetilde{\Sigma}$ with $\kappa_{+}(Y)<\delta$. Then, $\Sigma^{\prime}$ is invariant and by Lemma 2.3, $\kappa_{+}(Z)<\delta$ for any irreducible component $Z$ of $\Sigma^{\prime}$. Therefore, we can find $m \geq 0$ and $1 \leq \delta_{1}<\delta$ such that $\kappa_{m n_{0}}(Z) \leq \delta_{1}^{m n_{0}}$ for any irreducible component $Z$ of $\Sigma^{\prime}$. Finally denote by $\Sigma^{\prime \prime}$ the union of the nonperiodic irreducible components of $\widetilde{\Sigma}$ and $l$ the number of irreducible components of $\Sigma^{\prime \prime}$.

Choose an integer $N$ large enough so that $M^{l+m} \max \left(\delta_{0}, \delta_{1}\right)^{N m n_{0}}<\delta^{N m n_{0}}$ where $M$ denotes the maximal value of $\kappa_{n_{0}}$ on $X$. It is enough to check the last property in the proposition for $n_{1}:=N m n_{0}$. For this purpose, we only have to show that $\kappa_{-n_{1}}\left(V_{0}\right)<\delta^{n_{1}}$ for any irreducible analytic set $V_{0}$ of dimension $q^{\prime} \geq q$ which intersects $\Omega \backslash \Sigma$. Consider such an analytic set $V_{0}$ and an arbitrary sequence $V_{-N m}, V_{-N m+1}, \ldots, V_{0}$ of irreducible analytic subsets of dimension $q^{\prime}$ of $X$ such that $V_{-i+1}=f^{n_{0}}\left(V_{-i}\right)$. Since $f^{-1}(\Omega \backslash \Sigma) \subset \Omega \backslash \Sigma$ and since $f$ is surjective, all these analytic sets intersect $\Omega \backslash \Sigma$. It is also clear that the considered sequence contains at most $l$ elements which are irreducible components of $\Sigma^{\prime \prime}$. We show that $\kappa_{N m n_{0}}\left(V_{-N m}\right)<\delta^{N m n_{0}}$ which implies that $\kappa_{-n_{1}}\left(V_{0}\right)<\delta^{n_{1}}$ and completes the proof. We distinguish three cases.

If $V_{0}$ is not an irreducible component of $\Sigma^{\prime}$ (in particular when $q^{\prime}>q$ ), the sequence of $V_{-n}$ does not contain any irreducible component of $\Sigma^{\prime}$. It contains at most $l$ elements which are irreducible components of $\Sigma^{\prime \prime}$. The other components intersect $\Omega \backslash \widetilde{\Sigma}$. Therefore, we have

$$
\kappa_{N m n_{0}}\left(V_{-N m}\right) \leq \prod_{n=0}^{N m-1} \kappa_{n_{0}}\left(V_{-N m+n}\right) \leq M^{l} \delta_{0}^{N m n_{0}}<\delta^{N m n_{0}} .
$$

From now on, we only have to consider the case where $q^{\prime}=q$.

If $V_{-N m}$ is an irreducible component of $\Sigma^{\prime}$ then $V_{-i}$ is an irreducible component of $\Sigma^{\prime}$ for $0 \leq i \leq N m$ since $\Sigma^{\prime}$ is invariant. It follows from properties of cocycles that

$$
\kappa_{N m n_{0}}\left(V_{-N m}\right) \leq \prod_{i=0}^{N-1} \kappa_{m n_{0}}\left(V_{-(N-i) m}\right) \leq \delta_{1}^{N m n_{0}}<\delta^{N m n_{0}} .
$$

Otherwise, let $0 \leq s \leq N-1$ be the largest integer such that $V_{-i m}$ is an irreducible component of $\Sigma^{\prime}$ for $i \leq s$. Then $V_{-n}$ is not an irreducible component of $\Sigma^{\prime}$ for $n \geq(s+1) m$. Therefore, since the considered sequence contains at most 
$l$ elements which are irreducible components of $\Sigma^{\prime \prime}$, we have

$$
\begin{aligned}
\kappa_{N m n_{0}}\left(V_{-N m}\right) & \leq \prod_{n=0}^{N m-s m-1} \kappa_{n_{0}}\left(V_{-N m+n}\right) \prod_{i=0}^{s-1} \kappa_{m n_{0}}\left(V_{-(s-i) m}\right) \\
& \leq \prod_{n=0}^{N m-(s+1) m-1} \kappa_{n_{0}}\left(V_{-N m+n}\right) \prod_{N m-(s+1) m}^{N m-s m-1} \kappa_{n_{0}}\left(V_{-N m+n}\right) \prod_{i=0}^{s-1} \kappa_{m n_{0}}\left(V_{-(s-i) m}\right) \\
& \leq M^{l} \delta_{0}^{[N m-(s+1) m] n_{0}} M^{m} \delta_{1}^{s m n_{0}}<\delta^{N m n_{0}} .
\end{aligned}
$$

This completes the proof.

Proof of Theorem 1.2. Fix a real number $\delta>1$. We apply Proposition 2.4 to $n_{0}:=1, q:=k-1$ and $\Omega=\Omega_{0}:=X$. Since $\min \kappa_{1}=1$, we can find an invariant hypersurface $\Sigma_{1}$ and an integer $n_{1}$ such that $\left\{\kappa_{-n_{1}} \geq \delta^{n_{1}}\right\} \backslash \Sigma_{1}$ is of dimension at most equal to $k-2$. Moreover, $\liminf \left[\kappa_{n}\right]^{1 / n} \geq \delta$ and $\lim \inf \left[\kappa_{-n}\right]^{1 / n} \geq \delta$ on $\Sigma_{1}$. The Zariski open set $\Omega_{1}:=X \backslash \Sigma_{1}$ satisfies $f^{-1}\left(\Omega_{1}\right) \subset \Omega_{1}$. Hence, we can apply Proposition 2.4 inductively on $q$. We find invariant analytic subsets $\Sigma_{k-q}$ of pure dimension $q, 0 \leq q \leq k-1$, such that $\liminf \left[\kappa_{n}\right]^{1 / n} \geq \delta$ and $\liminf \left[\kappa_{-n}\right]^{1 / n} \geq \delta$ on $\Sigma_{q}$. Moreover, if $\Omega_{k-q}:=X \backslash \Sigma_{1} \cup \ldots \cup \Sigma_{k-q}$, then $\left\{\kappa_{-n_{k-q}} \geq \delta^{n_{k-q}}\right\} \cap \Omega_{k-q}$ is of dimension at most equal to $q-1$ for some integer $n_{k-q}$.

When $q=0$ the last intersection is empty. We deduce that $\kappa_{-n_{k}}<\delta_{0}^{n_{k}}$ on $\Omega_{k}$ for some constant $\delta_{0}<\delta$. This, the fact that $f^{-1}\left(\Omega_{k}\right) \subset \Omega_{k}$ together with the properties of cocycles imply that $\kappa_{-m n_{k}} \leq \delta_{0}^{m n_{k}}$ on $\Omega_{k}$ for $m \geq 0$. By Lemma 2.2, $\lim \sup \left[\kappa_{-n}\right]^{1 / n} \leq \delta_{0}$ on $\Omega_{k}$.

If $x$ is an arbitrary point in $X$, define $\kappa_{-}(x):=\liminf \left[\kappa_{-n}(x)\right]^{1 / n}$. When $\kappa_{-}(x)<\delta, x$ belongs to $\Omega_{k}$. Hence, $\lim \sup \left[\kappa_{-n}(x)\right]^{1 / n} \leq \delta_{0}<\delta$. This holds for any $\delta>\kappa_{-}(x)$. We deduce that $\left[\kappa_{-n}(x)\right]^{1 / n}$ converge to $\kappa_{-}(x)$. We also obtain from the previous analysis that $\left\{\kappa_{-} \geq \delta\right\}$ is equal to $\Sigma_{1} \cup \ldots \cup \Sigma_{k}$. By Proposition 2.4. this set is contained in the orbit of $\left\{\kappa_{n} \geq \delta^{n}\right\}$ for every $n \geq 0$.

Proof of Corollary 1.3. Let $x$ be a point in $X$. Assume that $x_{m}:=f^{m n_{k}}(x)$ is in $\Omega_{k}$ for every $m \geq 0$. We have $\kappa_{-n_{k}}\left(x_{m}\right) \leq \delta_{0}^{n_{k}}$ for every $m \geq 0$ and hence $\kappa_{n_{k}}\left(x_{m-1}\right) \leq \delta_{0}^{n_{k}}$ for every $m \geq 1$. Since $\left(\kappa_{n}\right)$ is sub-multiplicative, $\kappa_{m n_{k}}(x) \leq$ $\delta_{0}^{m n_{k}}$ for every $m \geq 1$. Lemma 2.1 implies that $\lim \sup \left[\kappa_{n}(x)\right]^{1 / n} \leq \delta_{0}<\delta$. The first assertion follows.

Now assume that $\left(\kappa_{n}\right)$ is multiplicative. Define $\kappa_{+}(x):=\liminf \left[\kappa_{n}(x)\right]^{1 / n}$. We have seen that if $x$ belongs to $\Sigma_{1} \cup \ldots \cup \Sigma_{k}$ then $\kappa_{+}(x) \geq \delta$. So, the properties of multiplicative cocycles imply that if $x$ is sent by a $f^{n}$ to a point in $\Sigma_{1} \cup \ldots \cup \Sigma_{k}$ then $\kappa_{+}(x) \geq \delta$. In other words, for any $\delta>\kappa_{+}(x)$ and $m \geq 0, x_{m}:=f^{m n_{k}}(x)$ is in $\Omega_{k}$. Therefore, we have $\limsup \left[\kappa_{n}(x)\right]^{1 / n} \leq \delta_{0}<\delta$. This implies that $\limsup \left[\kappa_{n}(x)\right]^{1 / n} \leq \kappa_{+}(x)$. Hence, $\left[\kappa_{n}(x)\right]^{1 / n}$ converge to $\kappa_{+}(x)$. The identity $\kappa_{+} \circ f=\kappa_{+}$is a consequence of the properties of multiplicative cocycles. 
Proposition 2.5. Let $\left(\kappa_{n}\right)$ be a sub-multiplicative cocycle as above. Let $x$ be a point in $X$. Then, for $l \geq 0$ large enough, $\left[\kappa_{n}\left(f^{l}(x)\right)\right]^{1 / n}$ converge to a constant $\kappa(x)$ which does not depend on $l$. In particular, $x$ is sent by an iterate of $f$ to the invariant analytic set $\left\{\kappa_{-} \geq \kappa(x)\right\}$.

Proof. Let $\chi_{l}:=\lim \sup _{n \rightarrow \infty}\left[\kappa_{n}\left(f^{l}(x)\right)\right]^{1 / n}$. We have $\chi_{l} \geq 1$. Inequalities (1) for $s=1$ imply that $\kappa_{n+1}\left(f^{l}(x)\right) \leq M \kappa_{n}\left(f^{l+1}(x)\right)$. Therefore, the sequence $\left(\chi_{l}\right)_{l \geq 0}$ is increasing. Define $\delta:=\lim _{l \rightarrow \infty} \chi_{l}=\sup _{l} \chi_{l}$. If $\delta=1$, we have $\chi_{l}=1$; hence $\left[\kappa_{n}\left(f^{l}(x)\right)\right]^{1 / n}$ converge to 1 for every $l \geq 0$. Assume now that $\delta>1$. We use the notations and the arguments in the proofs of Theorem 1.2 and Corollary 1.3. When $l$ is large enough, we have $\chi_{l}>\delta_{0}$. Therefore, $f^{l}(x)$ is sent by an iterate of $f$ to a point in $\Sigma_{1} \cup \ldots \cup \Sigma_{k}$. So, for $l$ large enough, $f^{l}(x)$ belongs to $\Sigma_{1} \cup \ldots \cup \Sigma_{k}$. For such an $l$, we have $\liminf _{n \rightarrow \infty}\left[\kappa_{n}\left(f^{l}(x)\right)\right]^{1 / n} \geq \delta$. This implies that $\left[\kappa_{n}\left(f^{l}(x)\right)\right]^{1 / n}$ converge to $\delta$. The last assertion in the proposition is deduced from Corollary 1.3 .

The following example shows that $\left[\kappa_{n}\right]^{1 / n}$ do not always converge when $n$ goes to infinity.

Example 2.6. Let $a$ be a fixed point of $f$ and $b$ a point in $f^{-1}(a) \backslash\{a\}$. Let $\lambda_{n}$ be positive numbers such that $\lambda_{n+1} \leq \lambda_{n}+1$. Define the analytic sub-multiplicative cocycle $\left(\kappa_{n}\right)$ by $\kappa_{n}(a):=e^{n}, \kappa_{n}(b):=e^{\lambda_{n}}$ and $\kappa_{n}(x):=1$ for $x \neq a, b$. Then, $\left[\kappa_{n}(b)\right]^{1 / n}$ converge if and only if the arguments of the complex numbers $n+i \lambda_{n}$ converge. It is not difficult to find $\lambda_{n}$ which do not satisfy this condition. In this case, the cocycle $\left(\kappa_{n}\right)$ is not multiplicative. When $\lambda_{n}=0,\left[\kappa_{n}\right]^{1 / n}$ converge but the limit is not an invariant function.

\section{Exceptional sets for meromorphic maps}

There are some natural cocycles over a complex dynamical system. We give some examples. Let $f: \mathbb{P}^{k} \rightarrow \mathbb{P}^{k}$ be a holomorphic map of algebraic degree $d \geq 2$ on the projective space of dimension $k$. It is induced by a map $F: \mathbb{C}^{k+1} \rightarrow \mathbb{C}^{k+1}$ whose irreducible components are homogeneous polynomials of degree $d$. The map $f$ defines a ramified covering of degree $d^{k}$ on $\mathbb{P}^{k}$. We refer to the survey [12] for the basic properties of $f$.

Let $\kappa_{n}(x)$ denote the multiplicity of $f^{n}$ at $x$, i.e. $f^{-n}(z)$ contains exactly $\kappa_{n}(x)$ points near $x$ for any $z$ generic close enough to $f^{n}(x)$. It is not difficult to check that $\left(\kappa_{n}\right)$ is an analytic multiplicative cocycle. We now give an application of Theorem 1.2.

Theorem 3.1 ([3, 5]). Let $f: \mathbb{P}^{k} \rightarrow \mathbb{P}^{k}$ be a holomorphic map of algebraic degree $d \geq 2$. Then there is a proper analytic subset $\mathscr{E}$ of $\mathbb{P}^{k}$, possibly empty, which is totally invariant by $f: f^{-1}(\mathscr{E})=f(\mathscr{E})=\mathscr{E}$, and is maximal: $\mathscr{E}$ contains all the proper analytic subsets $E$ of $\mathbb{P}^{k}$ satisfying $f^{-m}(E) \subset E$ for some $m \geq 1$. 
Proof. We use the multiplicative cocycle $\left(\kappa_{n}\right)$ defined above. Let $E$ be as in the theorem. The sequence of analytic sets $\left(f^{-n m}(E)\right)$ is decreasing. Hence, there is an $n$ such that $f^{-n m-m}(E)=f^{-n m}(E)$. Since $f^{n m}$ is surjective, we deduce that $f^{-m}(E)=E$ and $f^{m}(E)=E$. Observe also that if $\widetilde{E}:=E \cup \ldots \cup f^{-m+1}(E)$ then $f^{-1}(\widetilde{E})=\widetilde{E}$. Replacing $E$ by $\widetilde{E}$ allows to assume that $m=1$ and that $E$ is totally invariant: $f^{-1}(E)=f(E)=E$. The union of the irreducible components of $E$, of a given fixed dimension, is also totally invariant. So, it is enough to consider the case where $E$ is of pure dimension $p$. Let $n_{0} \geq 1$ be an integer such that $f^{n_{0}}$ fixes all the irreducible components of $E$.

Denote by $g$ the restriction of $f^{n_{0}}$ to $E^{\prime}$, an irreducible component of $E$. We claim that the topological degree of $g$ is equal to $d^{p n_{0}}$, that is, $g$ defines a ramified covering of degree $d^{p n_{0}}$. Indeed, if $\omega$ is a Kähler form on $\mathbb{P}^{k}$, then $\left(f^{n_{0}}\right)^{*}\left(\omega^{p}\right)$ is

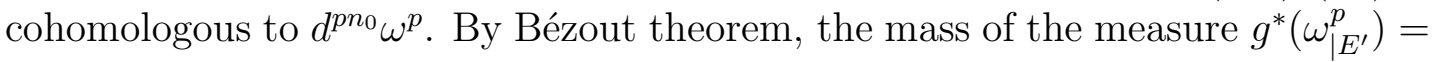
$\left(f^{n_{0}}\right)^{*}\left(\omega^{p}\right)_{\mid E^{\prime}}$ is equal to $d^{p n_{0}}$ times the mass of $\omega_{\mid E^{\prime}}^{p}$. This implies the claim.

Now, observe that the topological degree of $f^{n_{0}}$ is $d^{k n_{0}}$ while the topological degree of $g$ is equal to $d^{p n_{0}}$. This implies that $\kappa_{-n_{0}} \geq d^{(k-p) n_{0}}$ on $E^{\prime}$ since $f^{-n_{0}}\left(E^{\prime}\right)=E^{\prime}$. The inequality still holds if we replace $n_{0}$ by a multiple of $n_{0}$. Therefore, $E^{\prime}$ is contained in $\left\{\kappa_{-} \geq d^{k-p}\right\}$ and $E$ is contained in $\mathscr{E}^{\prime}:=\left\{\kappa_{-} \geq d\right\}$. Since $E$ is totally invariant, it is contained in $\mathscr{E}:=\cap_{n \geq 0} f^{-n}\left(\mathscr{E}^{\prime}\right)$. As above, we prove that $\mathscr{E}$ is totally invariant. This completes the proof.

The above result still holds for the restriction of $f$ to an invariant irreducible analytic set $X$ of dimension $p$. One lifts $f$ to a normalization of $X$. The map we obtain is a ramified covering of degree $d^{p}$. The cocycle $\left(\kappa_{n}\right)$ over this map can be defined as above. A simple decreasing induction on dimension allows to prove that $f$ admits a finite number of totally invariant analytic subsets. Moreover, the convex set of totally invariant probability measures, i.e. probability measures $\nu$ such that $f^{*}(\nu)=d^{k} \nu$, is of finite dimension. The equilibrium measure $\mu$ is the only totally invariant probability measure which has no mass on $\mathscr{E}$. The exceptional set $\mathscr{E}$ is also characterized by the property that $x \notin \mathscr{E}$ if and only if $d^{-k n}\left(f^{n}\right)^{*}\left(\delta_{x}\right)$ converge to $\mu$ [3, 5, see also [1, 9]. Here, $\delta_{x}$ denotes the Dirac mass at $x$.

Consider now a more general case. Let $(X, \omega)$ be a compact Kähler manifold of dimension $k$ and let $f: X \rightarrow X$ be a dominant meromorphic map. So, $f$ is a holomorphic map from $X \backslash I$ to $X$ where $I$ is the set of indeterminacy which is analytic and of codimension $\geq 2$. The closure $\Gamma$ of the graph of $f: X \backslash I \rightarrow X$ in $X \times X$ is an analytic set. If $\pi_{i}: X \times X \rightarrow X, i=1,2$, are the natural projections, then $f$ is equal to $\pi_{2} \circ\left(\pi_{1 \mid \Gamma}\right)^{-1}$ on $X \backslash I$. Define for $A \subset X$

$$
f(A):=\pi_{2}\left(\pi_{1}^{-1}(A) \cap \Gamma\right) \quad \text { and } \quad f^{-1}(A):=\pi_{1}\left(\pi_{2}^{-1}(A) \cap \Gamma\right) .
$$

Define also $f^{n}:=f \circ \cdots \circ f, n$ times, on a suitable dense Zariski open set and extend it to a meromorphic map on $X$. In general, we do not have $f^{2}(A)=$ $f(f(A))$ nor $f^{-2}(A)=f^{-1}\left(f^{-1}(A)\right)$. 
The set of indeterminacy $I$ is the set of points $x$ such that $f(x)$ is of dimension at least equal to 1 . Define $I_{1}:=I$ and $I_{n+1}:=f^{-1}\left(I_{n}\right)$ for $n \geq 1$. The orbit of a point $x$

$$
x, f(x), f^{2}(x), \ldots, f^{n}(x), \ldots
$$

is well-defined if $x \notin \cup_{n \geq 1} I_{n}$.

Let $I^{\prime}$ denote the set of points $x$ such that $f^{-1}(x)$ is of dimension larger than or equal to 1 . This is also an analytic subset of codimension $\geq 2$ of $X$. The fibers $f^{-1}(x)$ for $x \notin I^{\prime}$, are finite and contain the same number of points counted with multiplicity. This number $d_{t}$ is called topological degree of $f$. If $\nu$ is a finite measure on $X$ having no mass on $I^{\prime}$, then $f^{*}(\nu)$ is well-defined by the formula

$$
f^{*}(\nu):=\left(\pi_{1}\right)_{*}\left(\left(\pi_{2 \mid \Gamma}\right)^{*} \nu\right) .
$$

If $\nu$ is positive, the mass of $f^{*}(\nu)$ is equal to $d_{t}$ times the mass of $\nu$. Define $I_{1}^{\prime}:=I^{\prime}$ and $I_{n+1}^{\prime}:=f\left(I_{n}^{\prime}\right)$ for $n \geq 1$. If $x \notin \cup_{n \geq 1} I_{n}^{\prime}$, then $f^{-n}(x)$ contains exactly $d_{t}^{n}$ points counting with multiplicity.

Choose a finite or countable union $\widetilde{I}$ of proper analytic subsets of $X$ containing $I \cup I^{\prime}$ such that if $\widetilde{X}:=X \backslash \widetilde{I}$ then $f^{-1}(\widetilde{X})=f(\widetilde{X})=\widetilde{X}$. So, $f: \widetilde{X} \rightarrow \widetilde{X}$ defines a "ramified covering" of degree $d_{t}$. We consider the topology on $\widetilde{X}$ induced by the Zariski topology on $X$. As in the case of holomorphic maps on $X$, one can define cocycles over $f: \widetilde{X} \rightarrow \widetilde{X}$. Theorem 1.2 and Corollary 1.3 still hold in this case.

Recall that the dynamical degree of order $p$ of $f, 0 \leq p \leq k$, is defined by

$$
d_{p}:=\lim _{n \rightarrow \infty}\left(\int_{X}\left(f^{n}\right)^{*}\left(\omega^{p}\right) \wedge \omega^{k-p}\right)^{1 / n} .
$$

Note that $\left(f^{n}\right)^{*}\left(\omega^{p}\right)$ is a $(p, p)$-form with $L^{1}$ coefficients. The last limit always exists and the dynamical degrees are bi-meromorphic invariants [4]. We also have $d_{0}=1$ and $d_{k}=d_{t}$, the topological degree of $f$.

Theorem 3.2. Let $f: X \rightarrow X$ be a dominant meromorphic map as above. Assume that the topological degree of $f$ is strictly larger than the other dynamical degrees. Then there is a proper analytic subset $\mathscr{E}$ of $X$, possibly empty, such that

1. all the irreducible components of $\mathscr{E}$ intersect $\widetilde{X}$;

2. $\mathscr{E}$ is totally invariant: $f^{-1}(\mathscr{E} \cap \widetilde{X})=f(\mathscr{E} \cap \widetilde{X})=\mathscr{E} \cap \widetilde{X}$;

3. $\mathscr{E}$ is maximal: if $E \subset X$ is a proper analytic subset whose irreducible components intersect $\widetilde{X}$ and if $f^{-m}(E \cap \widetilde{X}) \subset E$ for some $m \geq 1$, then $E \subset \mathscr{E}$

One can also characterize $\mathscr{E}$ by the following property. For $x$ a point in $\widetilde{X}$, the sequence of probability measures $d_{t}^{-n}\left(f^{n}\right)^{*}\left(\delta_{x}\right)$ converges to the equilibrium measure of $f$ if and only if $x \notin \mathscr{E}$, see [2, 5, 10] for the proof. 
The proof of Theorem 3.2 follows the arguments in Theorem 3.1. We only need the following lemma.

Lemma 3.3. Let $E$ be a proper irreducible analytic subset of $X$ which intersects $\widetilde{X}$ and $n_{0} \geq 1$ an integer such that $f^{n_{0}}(E \cap \widetilde{X}) \subset E$. Then the topological degree of the meromorphic map $f^{n_{0}}: E \rightarrow E$ is at most equal to $\delta^{n_{0}}$ where $0<\delta<d_{t}$ is a constant independent of $E$ and $n_{0}$.

Proof. Denote by $g$ the map $f^{n_{0}}: E \rightarrow E$. Let $p$ be the dimension of $E, d$ the topological degree of $g$ and $\delta<d_{t}$ a constant larger than or equal to the dynamical degrees $d_{0}, \ldots, d_{k-1}$. The mass of $g^{*}\left(\omega_{\mid E}^{p}\right)$ is equal to $d$ times the mass of $\omega_{\mid E}^{p}$. We will bound the mass of $g^{*}\left(\omega_{\mid E}^{p}\right)$. Fix a constant $\epsilon>0$. In what follows, all the constants are independent of $E, n_{0}$ and $n$.

The form $\left(f^{n_{0}}\right)^{*}\left(\omega^{p}\right)$ has $L^{1}$ coefficients and is smooth on the Zariski open set $X \backslash \cup_{1 \leq n \leq n_{0}} I_{n}$ which contains $\widetilde{X}$. It also defines a positive closed $(p, p)$-current on $X$ whose mass is given by the formula

$$
\left\|\left(f^{n_{0}}\right)^{*}\left(\omega^{p}\right)\right\|:=\int_{X}\left(f^{n_{0}}\right)^{*}\left(\omega^{p}\right) \wedge \omega^{k-p} .
$$

Therefore, there is a constant $c>0$ such that this mass is bounded by $c\left(d_{p}+\epsilon\right)^{n_{0}}$. We regularize $\left(f^{n_{0}}\right)^{*}\left(\omega^{p}\right)$ using the results in [4]. There are smooth positive closed $(p, p)$-forms $\Omega_{i}^{ \pm}$such that $\Omega_{i}^{+}-\Omega_{i}^{-}$converge to $\left(f^{n_{0}}\right)^{*}\left(\omega^{p}\right)$ in the sense of currents and

$$
\left\|\Omega_{i}^{ \pm}\right\| \leq A\left\|\left(f^{n_{0}}\right)^{*}\left(\omega^{p}\right)\right\| \leq A^{\prime}\left(d_{p}+\epsilon\right)^{n_{0}}
$$

for some constants $A$ and $A^{\prime}$. Since $\left(f^{n_{0}}\right)^{*}\left(\omega^{p}\right)$ is smooth in a neighbourhood of $\widetilde{X}$, the construction in [4] gives forms $\Omega_{i}^{ \pm}$such that $\Omega_{i}^{+}-\Omega_{i}^{-}$converge uniformly on compact subsets of $\widetilde{X}$ to $\left(f^{n_{0}}\right)^{*}\left(\omega^{p}\right)$. It follows that

$$
\left\|g^{*}\left(\omega_{\mid E}^{p}\right)\right\|=\left\|\left(f^{n_{0}}\right)^{*}\left(\omega^{p}\right)_{\mid E \cap \tilde{X}}\right\| \leq \limsup _{i \rightarrow \infty} \int_{E} \Omega_{i}^{+} .
$$

The last integral depends only on the cohomology class of $\Omega_{i}^{+}$. The above bound on the mass of $\Omega_{i}^{+}$induces a bound on its cohomology class. Therefore, $\left\|g^{*}\left(\omega_{\mid E}^{p}\right)\right\| \leq A^{\prime \prime}\left(d_{p}+\epsilon\right)^{n_{0}}$ for some constant $A^{\prime \prime}$. The estimate also holds for the iterates of $g$ : we have $\left\|\left(g^{n}\right)^{*}\left(\omega_{\mid E}^{p}\right)\right\| \leq A^{\prime \prime}\left(d_{p}+\epsilon\right)^{n n_{0}}$. It follows that $d^{n} \lesssim\left(d_{p}+\epsilon\right)^{n n_{0}}$. Hence, $d$ is at most equal to $d_{p}^{n_{0}}$. This completes the proof.

\section{References}

[1] Briend J.-Y., Duval J., Deux caractérisations de la mesure d'équilibre d'un endomorphisme de $\mathrm{P}^{k}(\mathbf{C})$, Publ. Math. Inst. Hautes Études Sci., 93 (2001), 145-159.

[2] Dinh T.-C., Suites d'applications méromorphes multivaluées et courants laminaires, J. Geom. Anal., 15 (2005), no. 2, 207-227. 
[3] Dinh T.-C., Sibony N., Dynamique des applications d'allure polynomiale, J. Math. Pures Appl., 82 (2003), 367-423.

[4] — Regularization of currents and entropy, Ann. Sci. École Norm. Sup., 37 (2004), 959-971.

[5] - Equidistribution towards the Green current for holomorphic maps, Ann. Sci. École Norm. Sup., to appear.

[6] Favre C., Dynamique des applications rationnelles, PhD thesis, Université ParisSud, Orsay, 2000.

[7] — Multiplicity of holomorphic functions, Math. Ann., 316 (2000), no. 2, 355378.

[8] Favre C., Jonsson M., Brolin's theorem for curves in two complex dimensions, Ann. Inst. Fourier, 53 (2003), no. 5, 1461-1501.

[9] Fornæss J.-E., Sibony N., Complex dynamics in higher dimension. II. Modern methods in complex analysis (Princeton, NJ, 1992), 135-182, Ann. of Math. Stud., 137, Princeton Univ. Press, Princeton, NJ, 1995.

[10] Guedj V., Ergodic properties of rational mappings with large topological degree, Ann. of Math. (2), 161 (2005), no. 3, 1589-1607.

[11] Hartshorne R., Algebraic geometry, Graduate Texts in Mathematics, No. 52, Springer-Verlag, New York-Heidelberg, 1977.

[12] Sibony N., Dynamique des applications rationnelles de $\mathbb{P}^{k}$, Panoramas et Synthèses, 8 (1999), 97-185.

[13] Walters P., An introduction to ergodic theory, Graduate Texts in Mathematics, 79, Springer-Verlag, New York-Berlin, 1982.

T.-C. Dinh, UPMC Univ Paris 06, UMR 7586, Institut de Mathématiques de Jussieu, F75005 Paris, France. dinh@math.jussieu.fr, http://www.math.jussieu.fr/ $\sim$ dinh 